\title{
Tri-plane Representation and Analysis in Transform Coding*
}

\author{
Feifei Zhou, Rui Yang, Bo Li \\ Beijing Key Laboratory of Digital Media, School of Computer Science and \\ Engineering, Beihang University, Beijing, 100191, China \\ ffzhou@cse.buaa.edu.cn
}

\begin{abstract}
Representation and coding of transform coefficients is a key factor in image compression. In this work, we introduce a coefficient representation model, called tri-plane. The proposed method is able to bring more kinds of symbols and significance states, which deeply develops the correlations among transform coefficients. By utilizing new symbols and states, more precise classification can be achieved, which directly determines the prediction coding performance. Experimental results show that tri-plane representation outperforms the traditional bit-plane form in entropy, which removes more redundancy among coefficients and obtains more potential to improve coding efficiency. We hope this work would motivate more research and application work along this direction.
\end{abstract}

Keywords- Tri-plane representation; Bit-plane; prediction coding; Image compression; Transform coefficients

\section{INTRODUCTION}

Bit-plane prediction coding is an effective method to represent and encode transform coefficients in image compression. Subband transform, especially wavelet transform, achieves great success in image coding applications. Transform accomplish sparse representation of image data, which makes big contribution to improve coding efficiency. After transform, there is still redundancy between coefficients. In order to achieve higher compression ratio, bit-plane prediction is widely used to remove the redundancy.

Some notable bit-plane coding algorithms are proposed, such as EBCOT[1], SPIHT[2], SPECK[3] in wavelet domain and AGU-MHV[3] in DCT domain. EBCOT is the representative algorithm based on bit-plane prediction, which builds context model to remove coefficient redundancy based on correlation. It avoids the rigid quadtree and zero-block structures used in SPIHT and SPECK, and can exploits the correlation more sufficiently and flexibly. EBCOT has been adopted as the core coding algorithm of new international image compression standard JPEG2000[5]. AGU-MHV utilized a bit-plane dynamical arithmetical coding with a sophisticated context modelling to reduce statistical redundancy of quantized DCT coefficients. And this method provides better compression than JPEG and other DCT-based techniques. Moreover, it outperforms JPEG2000 and other wavelet-based image coders. In a word, these methods achieve high compression efficiency by using bit-plane prediction.

Meanwhile, statistics analyses of transform coefficients based on bit-plane have been reported. [6-9] discuss some models to describe the dependencies between coefficients based on bit-plane, which are helpful to improve compression performance. However, it is the fact that there is no breakthrough at the performance of bit-plane prediction coding in recent years. And the correlations between coefficients based on bit-plane seem to be utilized adequately. It is urgent to solve the problem that is how to explore the dependencies between coefficients further and how to use them to improve coding efficiency.

This paper proposed a new approach to represent transform coefficient, called tri-plane. Here, every coefficient is represented in form of trinary number. So triplane is produced, which will bring more symbol varieties and significance states. Then tri-planes of transform coefficients are characterized and analyzed. The results show the potential of tri-plane in image representation and coding.

The rest of paper is organized as follows. Section 2 analyzes the characteristic of bit-plane prediction coding, and summarizes the goal of coefficient representation. Section 3 describes tri-plane representation of transform coefficient, statistics and experimental results are also given in this section. Section 4 outlines the application of triplane, such as in image coding. The last section draws the conclusion and points the future work.

\section{AnAlysis OF Bit-Plane Prediction CODING}

To achieve high compression efficiency, appropriate representation and prediction coding of transform coefficients are essential approach. After transform, there is still redundancy between coefficients, which can be utilized to improve coding efficiency. Bit-plane prediction coding is widely used to remove the redundancy and achieve high compression efficiency.

\section{A. Bit-plane Representation}

In order to develop the correlation between coefficients, bit-plane representation of coefficients is generally adopted[5], as Fig. 1 shows. There, every coefficient is represented in the form of binary number. Then, the bits corresponding to same power compose a plane. So the

\footnotetext{
* This work was partially supported by the National 863 Program (2009AA12Z112) and the 973 Program (2010CB327900). The research was made in the State Key Laboratory of Virtual Reality Technologies and Systems.
} 
higher bit-plane is associated with higher power, which is more significant in sense of rate-distortion. During the coding procedure, the bit-plane is scanned from top to bottom.

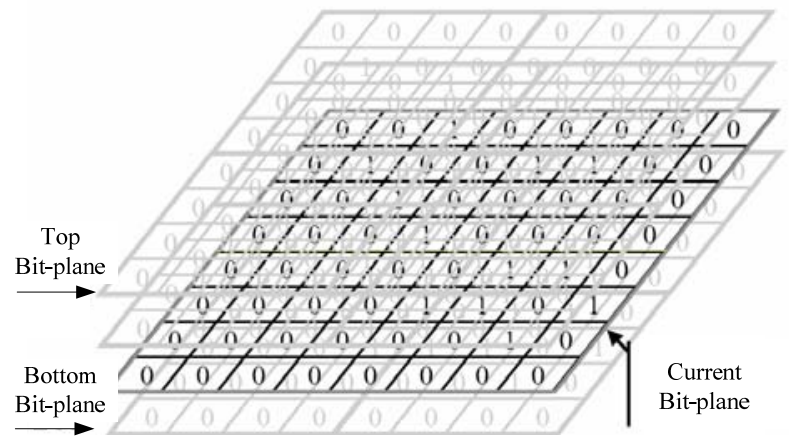

Fig. 1 Bit-plane Representation of Coefficients

By the representation of bit-plane, more correlations could be utilized. Transform removes redundancy between image pixels in space domain efficiently. But there is still correlation between transform coefficients. In transform domain, the neighbor coefficients are produced by neighbor image blocks of space domain. Generally, the adjacent image blocks appear in similar gray values, so magnitudes of neighbor coefficients are also close. Moreover, corresponding coefficients between sub-bands are associated to same image block, which also possess correlation. Binary representation makes it be possible to utilize these correlations in bit-plane levels, which is more refined than the whole coefficient representation. So most of prediction coding are based on bit-plane representation.

\section{B. Prediction Coding}

Prediction coding of coefficient bits makes compression approach high-order entropy of data. Prediction based on bit-plane utilized the encoded coefficients to evaluate the probability distribution of current bit to encode. The bits, which appear in similar probability distributions, will be classified into the same class. Then they are sent to same arithmetic coder to accomplish entropy coding. So prediction is a classification procedure, which is described in Fig.2. There, bit stream is sent to predictor, which is produced by scanning bit-plane in sequence.

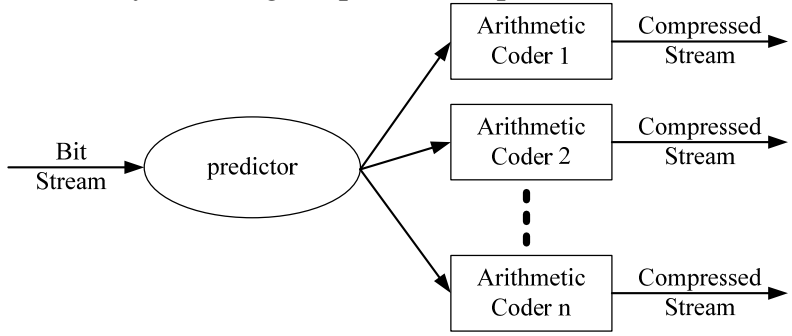

Fig. 2 Prediction Coding

Arithmetic coder is an indispensable part of high performance data compression, which theoretically could encode data close to Shannon entropy. To achieve high coding efficiency, image compression generally adopts arithmetic coding as the last step. By scanning and predicting based on bit-plane, the binary stream is classified into several sub-streams. Subsequently, every sub-stream is sent to one arithmetic coder, which removes the statistical redundancy of the bit stream fully.

\section{Intention of Representation}

The approach to represent coefficients directly affects the following coding. First, symbols to represent coefficients are the input source of prediction coding. Second, symbol kinds determine the potential states of each coefficient, which would produce different classification. Finally, coefficient representation also can be regarded as a classification procedure, which could change correlations between coefficients. So how to represent coefficients is a question deserved to discuss.

As the basis of prediction coding, appropriate representation should be studied in following aspects.

1) Correlation: Appropriate representation makes the following prediction coding be able to utilize the correlation between coefficients adequately. If granularity is too coarse, many correlations can not be utilized efficiently, such as whole coefficient coding. Reversely, bit-plane representation makes the correlations be able to utilize and develop fully.

2) Classification: Enough symbol varieties and states should be provided, which make it possible to carry out more precise classification. Good classification can distinguish the symbols with different probability distributions. However, this does not mean that symbol varieties and states should be as more as possible.

3) Entropy: Entropy of data source directly determines the ability to be compressed. Different representation can obtain different entropy. Shannon entropy is zero-order entropy of data source, which could indicate potential to be compressed. Though prediction coding could approach high-order entropy, Shannon entropy still can be regarded as reference guideline.

In a word, good representation should be helpful to develop more correlations between coefficients and distinguish different probability distributions. Besides, low entropy property is also a requirement for it.

\section{ANALYSIS OF TRI-PLANE}

Compared with bit-plane representation, tri-plane coding appears more potential in utilizing correlation and reducing redundancy. In this section, more analysis and experiments on tri-plane will be exhibited.

\section{A. Symbols and States}

Tri-plane representation introduces more varieties of symbols. Each plane is composed of symbol "0", " 1 " and "2". As bit-plane prediction coding, coefficients based on tri-plane representation are also classifies into three kinds: significance symbol (Sig. Sym. for short), refinement bit (Ref. Sym. for short) and sign bit, shown in Fig.3. Significance symbol and refinement symbol are used to 
represent the magnitude of the coefficient. Sign bit is used to indicate the coefficient is positive or negative. In the sense of rate-distortion, these three kinds of symbols have different significance. Given the same target bit rate, encoding more significance bits preferentially can improve the quality of reconstructed image.

\begin{tabular}{|c|c|c|c|c|c|c|c|}
\hline \multirow[b]{3}{*}{ Sig. Sym. } & 0 & 0 & 0 & 0 & 0 & 1 & 0 \\
\hline & 0 & 0 & $\mathbf{0}$ & $\mathbf{0}$ & $\mathbf{0}$ & $\mathbf{0}$ & 2 \\
\hline & 0 & $\mathbf{0}$ & 2 & $\mathbf{0}$ & 1 & $\mathbf{0}$ & $\mathbf{0}$ \\
\hline \multirow{6}{*}{$\underset{\text { Ref. Sym. }}{\longrightarrow}$} & 0 & $\mathbf{0}$ & $\mathbf{0}$ & 1 & $\mathbf{0}$ & 1 & 1 \\
\hline & 0 & 1 & $\mathbf{0}$ & 2 & 1 & $\mathbf{0}$ & 2 \\
\hline & 0 & $\mathbf{0}$ & 1 & 1 & $\mathbf{0}$ & 1 & $\mathbf{0}$ \\
\hline & 1 & $\mathbf{0}$ & $\mathbf{0}$ & $\mathbf{0}$ & 2 & $\mathbf{0}$ & 1 \\
\hline & . & . & . & . & . & . & . \\
\hline &. & $\cdot$ & . & . & $\dot{.}$ & $\dot{.}$ & . \\
\hline Sign bit & - & + & - & - & + & + & - \\
\hline
\end{tabular}

Fig. 3 Symbol Varieties of Tri-plane

As increasing of symbol varieties, every coefficient appears more significance states. Table I lists symbol and state comparison between bit-plane and tri-plane representation. States are classified into two kinds, called significant state (Sig. in Table I) and non-significant state (Non-Sig. in Table I). Significant state means that Sig. Sym. "2" or " 1 " appears in current encoding plane, or Sig. Sym. " 2 " or " 1 " has appeared above this plane (omitted in Table I). Reversely, non-significant state means that all encoded symbols are Sig. Sym. “0”. New symbol varieties expanded coefficient states. These are helpful to gain more precise prediction and classification of coefficients.

TABLE I Symbols and States Comparison

\begin{tabular}{|c|c|c|c|c|}
\hline & \multicolumn{2}{|c|}{ Bit-plane } & \multicolumn{2}{|c|}{ Tri-plane } \\
\hline & State & Symbol & State & Symbol \\
\hline \multirow{3}{*}{$\begin{array}{l}\text { Above } \\
\text { Plane }\end{array}$} & \multirow{2}{*}{ Sig. } & \multirow{2}{*}{ " $1 "$} & \multirow{2}{*}{ Sig. } & "2" \\
\hline & & & & “1” \\
\hline & Non-Sig. & “0” & Non-Sig. & “0” \\
\hline \multirow{3}{*}{$\begin{array}{l}\text { Current } \\
\text { Plane }\end{array}$} & \multirow{2}{*}{ Sig. } & \multirow{2}{*}{ “ 1 " } & \multirow{2}{*}{ Sig. } & "2" \\
\hline & & & & "1" \\
\hline & Non-Sig. & “0” & Non-Sig. & "0" \\
\hline
\end{tabular}

\section{B. Classification}

More symbol varieties and states of tri-plane make it possible to carry out more precise classification. Tri-plane representation introduces new symbol Sig. "2", which increases the significant states. However, it is meaningful when the new symbol and state can distinguish symbols with different probability distributions.

Hence, we administer a performance test for new symbol. For $512 \times 512 \times 8$ bit gray scale image “city”, after 6 level dyadic wavelet transform(Daubechies 9/7 filter is employed), probability distribution of current encoding symbol " $C$ " in HL direction is given in Table II, when the prediction coefficient "N1"(the one just above current encoding coefficient) corresponds to different symbol values. Here, only significance symbols are accounted, and omitting the subband which only includes symbol " 0 ”. The probability distributions are so different that more precise prediction classification can be achieved by utilizing new symbol.

TABLE II Probability Distribution

\begin{tabular}{|c|c|c|c|c|}
\hline \multirow{2}{*}{} & & \multicolumn{3}{|c|}{ Current plane of "N1" } \\
\cline { 3 - 5 } & & “0” & “1” & “2” \\
\hline \multirow{2}{*}{$\begin{array}{c}\text { Current } \\
\text { Symbol } \\
\text { "C” }\end{array}$} & “0” & $87 \%$ & $56 \%$ & $46 \%$ \\
\cline { 2 - 5 } & “1” & $10 \%$ & $29 \%$ & $32 \%$ \\
\cline { 2 - 5 } & “2” & $3 \%$ & $15 \%$ & $22 \%$ \\
\hline
\end{tabular}

\section{Entropy}

Entropy is the direct way to evaluate coding potential. Prediction coding can approach high-order entropy of data source. And different prediction coding algorithm could calculate to get different high-order entropy. Whereas, Shannon entropy (zero-order entropy) still can be treated as standard.

In order to evaluate performance of tri-plane representation, entropy experiments were carried out on many $512 \times 512 \times 8$ bit gray scale images. Daubechies $9 / 7$ filter is also employed to perform 6 level dyadic wavelet transform, and then wavelet coefficients are rounded. Next, coefficients are scanned and calculated entropy based on bit-plane or tri-plane. Entropy is defined as

$$
H(X)=N \cdot \overline{H(X)}=N \cdot \sum_{i=0}^{k} p(i) \log _{2} p(i)
$$

Where $N$ denotes the number of symbols, and $k$ denotes varieties of symbols. The results are given in Table III. Because entropy of sign bits is not changed when coefficients are represented in tri-plane, results are the statistic of Sig. Symbols and Ref. Symbols. And Table IV lists average entropy of every coefficient.

TABLE III Entropy of All Planes (bits)

\begin{tabular}{|c|c|c|c|}
\hline Images & Bit-plane & Tri-plane & Reduction \\
\hline Lena & 1536985 & 1409468 & 127517 \\
\hline Barbara & 1732753 & 1533125 & 199628 \\
\hline Z3 & 1555174 & 1464533 & 90641 \\
\hline Mexico & 1567574 & 1388194 & 179380 \\
\hline Tokyo & 1732488 & 1616225 & 116263 \\
\hline City & 2145086 & 2050261 & 94825 \\
\hline
\end{tabular}

TABLE IV Average Entropy of Every Coefficient (bits)

\begin{tabular}{|c|c|c|}
\hline Images & Bit-plane & Tri-plane \\
\hline Lena & 5.86 & 5.38 \\
\hline Barbara & 6.61 & 5.89 \\
\hline Z3 & 5.93 & 5.59 \\
\hline Mexico & 5.98 & 5.30 \\
\hline Tokyo & 6.61 & 6.17 \\
\hline City & 8.18 & 7.82 \\
\hline
\end{tabular}

Tri-plane representation outperforms than bit-plane form in entropy, which illuminates its potential to gain high coding efficiency. Generally, image corresponding to lower entropy is easier. But entropy changes caused by tri-plane do not show completely monotonicity. Probably, different 
representation changes correlation between different planes, which affects prediction coding further.

\section{APPLICATIONS}

Through entropy comparison, it is obvious that tri-plane possesses potential to promote performance. Pilot study on tri-plane application is attempted. Without adjusting prediction model[10], image coding algorithm based on triplane achieve nearly comparative result to JPEG2000[5], which is based on bit-plane. By optimizing prediction model, compression performance will improve.

Tri-plane prediction coding (even higher scale representation) will expand the application of image coding. Tri-plane representation enriches the varieties of symbols, which would affect classification and improve performance. Moreover, coefficients representation in other fields also can be attempted in this new form.

\section{CONCLUSION}

The major contribution of this work is proposing a concept, called tri-plane. This new representation form brings more symbol varieties and states, which could be combined and utilized to enrich following prediction classification. Tri-plane representation brings different correlation for coefficients, which would be used to improve coding efficiency.

This paper validates efficiency of tri-plane representation by calculating the zero-order entropy. Then there is still more work to do in the future. Coefficient representation is the primary step. Next, how to utilize symbols and states of tri-plane to construct efficient prediction model is still important issue.

\section{REFERENCES}

[1] D. Taubman, "High performance scalable image compression with EBCOT”, IEEE Trans. on Image Process., vol. 9, no. 7, pp. 11581170, 2000.

[2] A. Said and W.A. Pearlman, "A new fast and efficient image codec based on set partitioning in hierarchical trees", IEEE. Trans. Circ. Syst. Video Tech., vol. 6, pp. 243-250, June 1996.

[3] W. A. Pearlman, A. Islam, N. Nagaraj, "Efficient, Low-Complexity Image Coding with A Set-Partitioning Embedded Block Coder", IEEE. Trans. Circ. Syst. Video Tech., vol. 14, no. 11, pp. 1219-1235, 2004.

[4] N. N. Ponomarenko, K. O. Egiazarian, V. V. Lukin, and J. T. Astola, "High-Quality DCT-Based Image Compression Using Partition Schemes”, IEEE Signal Process. Lett., vol. 14, no.2, pp. 105-108, Feb. 2007.

[5] David S. Taubman, Michael W. Marcellin. JPEG2000 Image Compression Fundamentals, Standards and Practice. Kluwer Academic Publishers, 2001.

[6] R. W. Buccigrossi, E. P. Simoncelli, "Image Compression via Joint Statistical Characterization in the Wavelet Domain”, IEEE Trans. On Image Process., vol. 8, no. 12, pp. 1688-1701, Dec. 1999.

[7] X. T. Liu, “A New Approach to Time-Frequency Analysis”, 2010 Data Compression Conference, pp. 540.

[8] E. Y. Lam, "Statistical modelling of the wavelet coefficients with different bases and decomposition levels”, IEE Proc. - Vision, Image and Signal Process., vol. 151, no. 3, pp. 203-206, June 2004.

[9] M. D. Gaubatz, S. S. Hemami, "Efficient Entropy Estimation Based on Doubly Stochastic Models for Quantized Wavelet Image Data", IEEE Trans. On Image Process., vol. 16, no. 4, pp. 967-981, Apr. 2007.
[10]B. Li, H. Wang, "Bit plane predicting image compression algorithm based on wavelet packet transform," Journal of Computers, vol. 22, no. 7, pp. 685-691, Jul. 1999 (in Chinese). 